34 E. Schmidt u. H. Roemer, Vork. kohlenst., fr. Fettsäuren i. pflanzl. Fetten.

gar keinen sichern Schluss auf den Gehalt des Präparats an metallischem Eisen.

Da mehrere Fabriken chemisch-pharmaceutischer Präparate ein Ferrum reductum mit mehr als 90\% metallischem Eisen liefern, so hielt man eine derartige Forderung an den Gehalt des officinellen Präparats für berechtigt.

\title{
Mittheilungen aus dem Universitäts-Laboratorium zu
}

\section{Halle a; $\mathbf{S}$.}

\author{
Von Prof. Ernst Schmidt.
}

\section{Ueber das Vorkommen kohlenstoffreicher, freier Fettsäuren in pflanzlichen Fetten.}

Von Ernst Schmidt und H. Roemer.

In der jüngsten Zeit hat zunächst von der Becke ${ }^{1}$ und später von Rechenberg ${ }^{2}$ auf das Vorkommen kleiner Mengen von freien Fettsäuren in den normalen pflanzlichen Fetten aufmerksam gemacht; als Ergänzung zu jenen Mittheilungen mag die nachstehende Notiz dienen, welche das Vorkommen relativ grosser Mengen kohlenstoftreicher, freier Fettsäuren in dem Fette der Kokkelskörner, der Muskatbutter und dem Lorbeerfette erörtern soll.

\section{Fett der Kokkelskörner.}

Bei Gelegenheit der Versuche, welche der eine von uns (R.) anstellte, um die in den Kokkelskörnern nach den Angaben von Pelletier und Couerbe ${ }^{3}$ und von Steiner enthaltenen Alkaloïde zu isoliren, wurde unsere Aufmerksamkeit auf die grossen Mengen eines weissen, talgartigen, stark sauer reagirenden Fettes gelenkt, welche sowohl beim Auspressen der zerkleinerten Fruchte, als auch beim Auskochen derselben mit salzsäurehaltigem Wasser resultirten. Dieses Fett ist schon wiederholt der Gegenstand von Untersuchungen gewesen; aus keiner der darüber vorliegenden Mittheilungen geht jedoch mit Sicherheit hervor, worin die Ursache der stark sauren

1) Zeitschr. f. anal. Ch. 19, 297.

2) Journ. f. pract. Ch. 132, 519.

3) Annal d. Ch. 10, 181.

4) Bot. Jahresb. 1879, 632 . 
E. Schmidt u. H. Roemer, Vork. kohlenst., fr. Fettsäuren i. pflanzl. Fetten. 35

Reaction desselben zu suchen ist. Zwar giebt Francis ${ }^{1}$ an, dass das Fett der Kokkelskörner ausser dem Glycerid der Stearophansäure auch freie Stearophansäure in geringer Menge enthalte, jedoch enthält die bezügliche Arbeit keinerlei experimentelle Beweise dieser Behauptung. Ebensowenig findet sich in der Literatur ein positiver Nachweis, dass die von Francis als Stearophansãure und von Crowder als Bassiasäure beschriebene Fettsäure des Kokkelskörnerfettes mit der Stearinsäure identisch ist. Die Mittheilungen von Heintz ${ }^{3}$ über die Identität von Stearophansäure und Stearinsäure beziehen sich nur auf die kohlenstoffreichste Fettsäure des Menschenfettes, welche von jenem Forscher anfänglich mit dem Namen Stearophansäure bezeichnet war.

Die Gesammtmenge an Fett, welche die von uns verarbeiteten Kokkelskörner enthielten, betrug 23,6\%, die Menge der darin vorkommenden freien Fettsäuren $9,2 \%$. Die Trennung der freien Fottsäuren von den gleichzeitig vorhandenen Glyceriden lässt sich leicht derartig bewerkstelligen, dass man die heisse alkoholische Lősung des Gemisches mit alkoholischer Baryumacetatlösung im Deberschusse versetzt und damit einige Zeit erwärmt. Der nach dem Erkalten gesammelte und hierauf getrocknete Niederschlag liefert nach wiederholtem Auskochen mit Petroleumäther alsdann einen Rückstand, welcher im Wesentlichen aus den Baryumsalzen der ursprünglich im freien Zustande vorhandenen, kohlenstoffreichen Fettsäuren besteht. Das aus diesen Baryumsalzen durch Kochen mit Salzsäure wieder abgeschiedene, etwa $39 \%$ des Rohfettes betragende Säuregemisch, schmolz bei $66-68^{\circ} \mathrm{C}$; ; dasselbe bestand somit wohl mit Wahrscheinlichkeit nahezu aus Stearinsäure (Schmelzpunkt $69,2^{\circ} \mathrm{C}$.). In der That gelang es auch ohne erhebliche Schwierigkeit nahezu die Gesammtmenge des Rohproductes durch Umkrystallisation aus Eisessig oder aus Alkohol, durch Destillation im Iuftverdünnten Raume (bei $100 \mathrm{Mm}$. Druck lag der Siedepunkt fast constant bei 286 bis $288^{\circ} \mathrm{C}$.) und durch partielle Fällung mittelst Baryumacetat nach der Methode von Heintz, in eine Säure überzuführen, welche sich durch ibre physikalischen und chemischen Eigenschaften als reine Stearinsâure kennzeichnete.

1) Chem. Centr.-Bl. 1842, 671.

2) Chem. Centr. - Bl. 1853, 2.

3) Kopp - Will, Jahresb. V, 516. 
36 E. Schmidt u. H. Roemer, Vork. kohlenst, fr. Fettsäuren i. pflanzl. Fetten.

Die Analyse der scharf bei $69,2^{\circ} \mathrm{C}$. schmolzenden Säure lieferte folgende Zahlen:

1) $0,2395 \mathrm{~g}$. Substanz ergab 0,6675 g. $\mathrm{CO}^{2}$ und $0,2783 \mathrm{~g} \cdot \mathrm{H}^{2} \mathrm{O}$.

2) 0,2287 - - $\quad$ - $0,6363-\mathrm{CO}^{2}-0,2630-\mathrm{H}^{2} \mathrm{O}$.

\begin{tabular}{|c|c|c|c|}
\hline \multirow{2}{*}{\multicolumn{2}{|c|}{$\begin{array}{l}\text { Berechnet für } \\
\mathrm{C}^{14} \mathrm{H}^{30} \mathrm{O}^{2}\end{array}$}} & \multicolumn{2}{|c|}{ Gefunden } \\
\hline & & 1. & 2. \\
\hline $\mathrm{C}$ & 76,05 & 76,01 & 75,87 \\
\hline $\mathrm{H}$ & 12,67 & 12,90 & 12,77 \\
\hline
\end{tabular}

Die Identität dieser im freien Zustande, sowohl in den Kernen, als auch in den Schalen der Kokkelskörner enthaltenen Säure mit der Stearinsäure hat der eine von uns (R.)' weiter durch einen Vergleich der beiderseitigen Baryumsalze und der Methyl- und Aethyläther constatirt. Das Gleiche gilt für die entsprechende, aus den Glyceriden der Kokkelskörner durch Verseifung abgeschiedene, von Francis als Stearophansäure, von Crowder als Bassiasäure bezeichnete Fettsäure.

Ausser freier Stearinsäure enthalten die Kokkelskörner noch geringe Mengen anderer kohlenstoffreicher, freier Fettsäuren, welche jedoch nicht vollständig von einander getrennt werden konnten. Durch partielle Fällung mit Baryumacetat und wiederholte Omkrystallisation der aus den einzelnen Fractionen abgeschiedenen Fettsäuren wurden im Wesentlichon zwei Producte erhalten, die trotz der Constanz ihrer Schmelzpunkte - das eine schmolz bei $56,5^{\circ} \mathrm{C}$, das andere bei $32^{\circ} \mathrm{C}$. - sich bei der weiteren Prüfung nicht als chemische Individuen erwiesen.

Als nahezu reine Stearinsäure ergab sich auch ein aus Kokkelskörnern dargestelltes Product, welches unter dem Namen „Menispermin " im Handel bezogen war. ${ }^{2}$ Dieses sogenannte Menispermin, in welchem wir, dem Namen nach zu urtheilen, ein Alkaloïd der Kokkelskőrner vermutheten, bildete eine perlmutterglänzende, blättrig krystallinische, bei $67-68^{\circ} \mathrm{C}$. schmelzende, stickstofffreie Masse, aus welcher durch Umkrystallisiren aus Eisessig oder aus Alkohol jedoch nur ein Körper resultirte, der in seinen Eigenschaften und in seiner Zusammensetzung genau mit der Stearinsäure übereinstimmte.

1) Inauguraldissertation Jena 1882.

2) Als wesentlich verschieden von obigem Menispermin stellte sich ein aus Menispermum canadense dargestelltes Product gleichen Namens heraus, welches mir kürzlich von Hrn. Dr. Schuchardt in Cörlitz übermittelt warde. S. 
E. Schmidt u. H. Roemer, Vork. kohlenst., fr. Fettsäuren i. pflanzl. Fetten. 37

Die Analysen des sogenannten Menispermins ergaben folgende Kahlen:

1) 0,225 g. Substanz lieferte 0,625 g. $\mathrm{CO}^{2}$ und 0,259 g. $\mathrm{H}^{2} \mathrm{O}$.

2) 0,2475 - - $\quad$ - $0,6895-\mathrm{CO}^{2}-0,2905-\mathrm{H}^{2} \mathrm{O}$.

Berechnet für

$\mathrm{C}^{18} \mathrm{H}^{86} \mathrm{O}^{3}$

Gefunden

C 76,05

1.2.

H $\quad 12,67$

75,75

75,97

12,78

13,04 .

Muskatbutter.

Aus käuflicher Muskatbutter können nach dem im Vorstehenden angedeutoten Verfahren ebenfalls $3-4 \%$ freier Fettsäuren isolirt werden, welche im Wesentlichen aus Myristicinsäure, gemischt mit einer geringen Menge einer dem Anschein nach mit der Stearinsăure identischen Säure, bestehen. Auf das Vorkommon ersterer Säure in den Muskatnüssen, und zwar in dem ätherischen Oele derselben, ist bereits von Flückige $\mathrm{r}^{1}$ aufmerksam gemacht worden.

Die Trennung der freien Rohfettsäuren der Muskatbutter geschah nach den Angaben von $\mathrm{Krafft}^{2}$ durch fractionirte Destillation im luftverdünnten Raume. Die Hauptmenge derselben ging hierbei, bei einem Drucke ron $100 \mathrm{Mm}$., gegen $248^{\circ} \mathrm{C}$. über, und nur erst gegen Ende der Destillation stieg der Siedepunkt bis gegen $285^{\circ} \mathrm{C}$. Der bei $248^{\circ} \mathrm{C}$. siedende Antheil des Destillats konnte ohne Schwierigkeit durch Umkrystallisation aus verdünntem Alkohol in ein bei $53-54^{\circ} \mathrm{C}$. schmelzendes Product übergeführt werden, dessen Eigenschaften und Zusammensetzung vollständig mit der der Myristicinsäure ilbereinstimmen.

Die Analyse dieser Säure lieferte folgende Zahlen:

1) $0,18 \%$ g. Substanz lieferte 0,4899 g. $\mathrm{CO}^{2}$ und 0,2012 g. $\mathrm{H}^{2} \mathrm{O}$.
2) 0,229 -
0,618 $\mathrm{CO}^{2}-0,258-\mathrm{H}^{2} \mathrm{O}$.

Berechnet für $\mathrm{C}^{14} \mathrm{H}^{28} \mathrm{O}^{2}$

Gefunden

C 73,68 1. 2.

H 12,28

73,40

73,60

12,28

12,51 .

Aus den höher siedenden Antheilen des Rohproductes konnte durch partielle Fällung mit Baryumacetat und Umkrystallisation der

1) N. Rep. d. Pharm. 23, 117.

2) Ber. d. d. chem. Ges. XII, 1688. 
38 E. Schmidt u. H. Roemer, Vork. kohlenst., fr. Fettsäuren i. pflanzl. Süuren.

aus den Finzelfractionen wieder abgeschiedenen Fettsäuren, eine bei etwa $68^{\circ} \mathrm{C}$. schmelzende Verbindung isolirt werden, welche nach ihren Eigenschaften und ihrer Zusammensetzung wohl als Stearinsăure anzusprechen sein dürfte.

Die Analyse dieser Säure ergab folgende Daten:

0,178 g. Substanz lieferte 0,494 g. ${ }^{-\mathrm{CO}^{2}}$ und 0,204 g. $\mathrm{H}^{2} \mathrm{O}$.

\begin{tabular}{|c|c|c|}
\hline $\begin{array}{r}\text { Bere } \\
\mathrm{C}^{1}\end{array}$ & $\begin{array}{l}\text { hnet für } \\
\mathrm{H}^{36} \mathrm{O}^{2}\end{array}$ & Gefunden \\
\hline $\mathrm{C}$ & 76,05 & 75,69 \\
\hline & 12,67 & 12,73 . \\
\hline
\end{tabular}

Ob in der Muskatbutter auch freie Fettsäuren vorkommen, welche dem Kohlenstoffgehalte nach zwischen der Myristicinsäure und der Stearinsäure liegen, haben wir nicht entscheiden können.

Lorbeerfett.

Das kăufliche, durch warmes Auspressen der Früchte von Laurus nobilis gewonnene Lorbeerfett, enthält nur sehr geringe Mengen freier Fettsäuren; wesentlich reicher daran ist dagegen die Masse, welche durch Ausziehen der getrockneten Lorbeeren mit heissem, starkem Alkohol, Abdestilliren des Lösungsmittels und Waschen des Rückstandes mit heissem Wasser resultirt. Die Hauptmenge der aus letzterem Producte nach dem im Vorstehenden angegebenen Verfahren isolirten freien Fettsäuren, deren Menge $2-3 \%$ von den angewendeten Lorbeeren betrug, destillirte bei einem Drucke von $100 \mathrm{Mm}$. zwischen 260 und $265^{\circ} \mathrm{C}$. über; erst gegen Ende der Destillation stieg der Siedepunkt auf etwa $290^{\circ} \mathrm{C}$. Trotz des ziemlich constanten Siedepunktes, welchen der Hauptantheil dieses Destillats zeigt, scheint derselbe doch aus einem Gemenge verschiedener Fettsäuren $\mathrm{zu}$ bestehen, deren vollständige Trennung bisher ohne Erfolg versucht wurde. Nach wiederholter fractionirter Fällung mittelst Baryumacetat und sehr häufigem Umkrystallisiren der hierdurch gewonnenen Einzelfractionen, resultirte schliesslich eine nicht unbeträchtliche Menge einer zwischen 60 und $62^{\circ} \mathrm{C}$. schmelzenden Säure, deren Analysen Zahlen lieferten, die nahezu mit der Zusammensetzung der Palmitinsäure übereinstimmen. Wir sind jedoch weit entfernt hiernach jene Säure schon als Palmitinsäure ansprechen zu wollen, umsomehr als wir häufig auffallend constant schmelzende Verbindungen unter den Händen hatten, die bei wiederholter partieller Fällung sich als Gemische verschiedener Individuen herausstellten. 